\title{
La gestión del capital humano en una unidad académica de educación superior
}

\author{
SUSAN CAROL CUENTAS MARTEL ${ }^{*}$ \\ Escuela de Graduados - Pontificia Universidad Católica del Perú \\ Recibido el 20-03-2017; primera evaluación el 07-06-2018; \\ segunda evaluación el 09-07-2018; aceptado el 10-07-2018
}

\section{Resumen}

La presente sociedad, caracterizada por el desarrollo incesante de la ciencia y del conocimiento, demanda, de las universidades, una renovación de la gestión, donde las personas que producen el conocimiento sean el centro; es decir, una gestión del capital humano. En ese sentido, este artículo presenta una investigación que analiza las estrategias de gestión del capital humano, con respecto a los docentes, en la Facultad de Educación de una universidad de Lima.

La metodología es mixta, cuantitativa y cualitativa. Se aplicó entrevistas y encuestas a directivos y docentes, respectivamente, y se obtuvo información sobre las estrategias dirigidas a valorar y potenciar el capital humano en la institución. Aunque los resultados evidenciaron que estas son insuficientes, se destaca el compromiso institucional de los docentes.

Palabras clave: gestión del conocimiento, educación superior, estrategias, capital humano.

\section{Management of human capital in higher education: analysis of a university}

\section{Abstract}

The present society, characterized by the unceasing development of science and knowledge, demands from the universities a renewal of management, where the people who produce knowledge are the center, that is, a human capital management. In this sense, this article presents an investigation that analyzes the

\footnotetext{
* Magíster en Gestión Educativa por la Pontificia Universidad Católica del Perú, y Magíster en Gerencia Social por la misma universidad. También es máster en Matrimonio y Familia por la Universidad de Navarra en Espańa. Ha realizado estudios en la universidad de Barcelona y en el Centro de Formación de la Cooperación Española en MontevideoUruguay. Actualmente, se desempeńa como docente universitaria y conferencista en temas de Educación Familiar y Gestión. Correo: susancarolc@gmail.com
} 
Strategies of Human Capital Management with respect to the teachers in the Faculty of Education of a university of Lima.

The methodology is mixed, quantitative and qualitative. Interviews and surveys were applied to managers and teachers, respectively, and information was obtained on strategies aimed at valuing and promoting human capital in the institution, although the results showed that these are insufficient, emphasizing the institutional commitment of teachers.

Keywords: Knowledge management, higher education, strategies, human capital.

\section{Gestão do capital humano no ensino superior: análise de uma universidade}

\section{RESUMO}

Esta sociedade caracterizada pelo contínuo desenvolvimento da ciência e do conhecimento, universidades exigem uma renovação da gestáo, onde as pessoas estão produzindo centro de conhecimento, ou seja, gestão de capital humano. Nesse sentido, este artigo apresenta pesquisa que analisa as estratégias de Human Capital Management sobre os professores da Faculdade de Educação de uma universidade em Lima.

A metodologia é mista, quantitativa e qualitativa. entrevistas e pesquisas de diretores e professores, respectivamente foi aplicado, e foi obtido informaçóes sobre estratégias para avaliar e melhorar o capital humano na instituição, embora os resultados mostraram que estas são insuficientes, o compromisso institucional de professores está.

Palavras-chave: gestão do conhecimento, o ensino superior, as estratégias, o capital humano

\section{INTRODUCCIÓN}

En la sociedad del conocimiento, la prioridad de las organizaciones educativas de calidad es el Know how de las personas y sus demás competencias y habilidades; es decir, el capital humano. Por ello, este se ha constituido en el capital más valioso que posee una organización. Esto adquiere mayor relevancia en la universidad, donde uno de sus fines es la producción y generación de nuevo conocimiento en beneficio de la sociedad.

En ese sentido, una gestión universitaria de calidad debería priorizar el capital humano con el que cuenta y planificar estrategias dirigidas a desarrollarlo; por ello, la gestión del capital humano en la universidad adquiere gran relevancia. 
Por lo mencionado, la investigación basó su estudio en el enfoque del capital humano y se acercó a la realidad de la gestión en las instituciones de educación superior. Para ello se planteó la pregunta: ¿Cómo se desarrollan las estrategias de gestión del capital humano con respecto a los docentes de la especialidad de Lengua y Literatura con diez a más años de antigüedad, en la Facultad de Educación de una universidad particular de Lima Metropolitana?

El objetivo general de la investigación fue identificar las estrategias de gestión del capital humano que se desarrollan en la institución. A partir de este objetivo, se plantearon los siguientes objetivos específicos:

- Identificar las estrategias de Gestión del Capital Humano de la Facultad de Educación que desarrollan los directivos con respecto a los docentes.

- Analizar las percepciones de los docentes con respecto a las estrategias de Gestión del Capital Humano desarrollada en la Facultad.

Como producto de la teoría referente al capital humano que sirvió de base para el estudio, se establecieron las categorías de análisis. La información obtenida se analizó a la luz de estas categorías que representaban las diferentes estrategias de gestión. Para ello, se siguió una metodología mixta: cuantitativa y cualitativa. La información tuvo, por un lado, tratamiento estadístico, y, por otro, tratamiento cualitativo. Finalmente, se trianguló la información, a fin de obtener resultados con un mayor grado de validez y confiabilidad.

La investigación es original en el campo de la educación universitaria del país, ya que los otros estudios provienen del nivel básico educativo o del campo empresarial. Asimismo, el desarrollo de este tipo de estudios contribuye en la mejora de la gestión universitaria y, por consiguiente, con el progreso de la educación superior en el país.

\section{FundaMeNTACIÓN TEÓRICA}

En el presente contexto, el papel predominante que ha adquirido el conocimiento como determinante principal de la productividad, competitividad y desarrollo de los países, ha obligado a los responsables de la educación a redefinir sus fines, bases teóricas de los planes de estudios, estructuras y formas en que imparte la educación, en general, su gestión.

En ese sentido, la reforma de la gestión en la universidad implica adoptar una gestión del conocimiento con la finalidad de afrontar con éxito el principal desafío de la Educación Superior en la actualidad: participar en la creación y la difusión de los conocimientos para resolver los problemas de la sociedad, y formar profesionales con ética donde se priorice el factor humano, como el recurso más valioso que posee una organización. 
[...] un elemento central en la nueva forma de gestionar es desarrollar la capacidad para identificar, producir, tratar, transformar, difundir y utilizar información a fin de crear y aplicar conocimientos necesarios que conduzcan al desarrollo humano con la finalidad de evitar los efectos perversos de la nueva cultura de la sobre información sin saber y, lo que es peor, sin pensar ni reflexionar. (Unesco, 2005, p. 21)

La gestión del conocimiento, aplicada a la universidad, es un nuevo paradigma para orientar los recursos científicos y tecnológicos de manera sistemática a fin de alcanzar los objetivos deseados por la institución y sociedad en general. Es, pues, una evolución de las ciencias y las tecnologías para saber de qué modo hay que formar especialistas y líderes con valores éticos capaces de contribuir a resolver los problemas de la presente sociedad y de sus instituciones en general (Pérez, 2011, p. 10). En este paradigma, la valorización de los recursos humanos con la formación del personal y la innovación permanente con la administración de los recursos técnicos son la clave para lograr la excelencia y eficiencia organizacional.

En ese sentido, el futuro exitoso de las universidades, que tienen la función de formar profesionales competentes, depende de su capacidad de convertir en ventaja su recurso más preciado: el capital humano. Por consiguiente, la universidad deberá ser capaz de potenciar y estimular todas las habilidades necesarias en los miembros de la institución que le permitan desarrollar una serie de habilidades y competencias destinadas a generar más conocimiento y que este repercuta en beneficio de la institución y, por consiguiente, del país. «People represent a vital resource of all organizations, whatever is their activity field, a resource which ensures survival, development and their competitiveness success» (Dădârlat y Dumitraşcu, 2015, p. 85).

Por lo tanto, una óptima gestión del capital humano es un aspecto trascendental que deben afrontar y superar eficientemente las universidades que deseen ser competitivas y exitosas; sobre todo, ser socialmente relevantes. Ello implica implementar mecanismos y procedimientos que aseguren la transformación del conocimiento de las personas en una ventaja corporativa y competitiva; es decir, ventaja de conocimiento que se constituye en la única ventaja comparativa sostenible (Sáez Vacas, García, Palao y Rojo, 2003).

La gestión del capital humano es un nuevo enfoque que considera a las personas como seres humanos dotados de un capital valioso. Las personas son, ahora, las nuevas socias de la organización, que aplican sus conocimientos y habilidades para garantizar el cumplimiento de los objetivos. En este nuevo enfoque, se persigue la optimización de las habilidades de las personas, su participación, creatividad y mejoramiento continuo. 
Por lo mencionado, en la investigación se tomó los aportes de diferentes especialistas en el tema, como Friedman, Hatch, Walker y Morales (2000), Davenport (2006), Alles (2006), entre otros, Puesto que, estos especialistas provienen del campo empresarial, sus aportes se adaptaron al campo de la educación.

La parte analítica del estudio se realizó a la luz del planteamiento de Daverport, pues las estrategias de gestión que plantea son más acordes al campo educativo y se presentan más flexibles para su adaptación en la educación superior.

Siguiendo a Davenport (2006), una eficiente gestión del capital humano conlleva el desarrollo de las siguientes estrategias:

1. Contratación de las personas adecuadas

2. Disposición del clima institucional

3. Constitución del capital humano

4. Retención del capital humano

La primera estrategia implica una contratación eficaz de inversores de capital humano. Para Davenport (2006), disponer del capital humano adecuado supondrá la mayor diferencia competitiva. En casos en que la institución no pueda mejorar las destrezas y el talento del capital humano, la contratación es la única solución. Los aspectos más relevantes de esta estrategia son la contratación y la consideración. La primera se refiere al contrato psicológico, que engloba la red de aspectos escritos y no escritos, como resultado de la interacción entre el docente que aspira a la plaza y el representante de la institución educativa, donde la duración y claridad son fundamentales para evitar la reducción del compromiso y de la inversión de capital humano. La segunda implica la correspondencia entre lo que invierte el docente (trabajo, dedicación) y lo que otorga el empleador como retribución (sueldo, incentivos económicos y o económicos).

La segunda estrategia, disposición del clima institucional, se refiere a los aspectos con los que debe contar el ambiente en la institución. En una organización educativa, el docente da su tiempo y despliega sus capacidades con la finalidad de recibir una retribución. En ese sentido, es importante identificar cuáles son los elementos que en el ambiente laboral hacen posible el rendimiento máximo del docente. Según Davenport (2006), el contexto de una inversión máxima de capital humano consta de 6 elementos: alineación con la estrategia institucional, el entendimiento y aceptación mutua, la competencia, la autonomía y el reforzamiento del ROIw.

La alineación se refiere a la traducción de objetivos en cada área de la organización, que cada docente conozca qué es lo que se espera lograr, y de 
qué manera él contribuye al logro de los objetivos institucionales. El segundo elemento, entendimiento y aceptación mutua implica que la institución brinde la información necesaria con respecto a las expectativas del docente, y cuáles serán los frutos de su inversión de capital humano. El tercer elemento, la competencia se refiere a la valoración y acciones que desarrolla la organización para perfeccionar las competencias de los docentes. El cuarto elemento, la autonomía, implica el grado de libertad que la institución otorga al docente para asumir nuevas responsabilidades y que funciona como aliciente para él. Finalmente, el ROIw en sus siglas en inglés (retorno de la inversión del capital humano) está compuesto por todos los incentivos requeridos para suscitar un incremento de la inversión del docente, vienen a ser la satisfacción intrínseca del docente con su empleo, la oportunidad de desarrollo profesional dentro de la institución y el reconocimiento de sus logros.

La tercera estrategia es la constitución del capital humano. Hace referencia a los procesos necesarios para lograr un capital humano competente. Estos son tres: el aprendizaje formal, el aprendizaje informal y la construcción del capital de información.

El aprendizaje formal engloba las actividades estructuradas, generalmente bajo la forma de un curso, con fechas y horarios y objetivos predeterminados. Es una transmisión de conocimientos y habilidades. Se caracteriza por ser organizada, planificada y evaluable (Alles, 2006). Por otro lado, el aprendizaje informal es la adquisición de conocimientos en el mismo puesto de trabajo, mediante equipos que se conforman voluntariamente o mediante otros medios no determinados. Por ejemplo, cuando un docente pregunta a un colega cómo se realiza determinada tarea, este aprendizaje es penetrante continuo y causal. «Organizations accumulate knowledge over time, learning from their members who simultaneously assimilate organizational beliefs» (Farace y Mazzotta, 2015, p. 43). Por último, el capital de información se refiere a la transformación de los conocimientos generados en la organización en capital de información, esto es, convertir el conocimiento tácito a explícito, pasarlo a un material tangible, un documento, un $\mathrm{CD}$ u otros y ponerlo a disposición de toda la comunidad educativa.

La cuarta estrategia, retención de capital humano, implica que los docentes no dejen la organización y se esfuercen de manera voluntaria, a fin de generar mayor rendimiento del capital humano. El docente comprometido trabaja en aras de la institución; por ello, recibe una recompensa y sigue actuando en interés de la organización sin dejarla. Es decir, recibe una retribución y reconocimiento por su esfuerzo y aporte. Al respecto, Sveiby (2000) manifiesta 
que los empleados suelen ser fieles a su empresa si se les trata correctamente y tienen la sensación de que comparten una responsabilidad.

El rendimiento intenso aporta una recompensa que, a su vez, incrementa la satisfacción. La satisfacción es dependiente del rendimiento. "La satisfacción laboral es importante porque las personas pasan una porción considerable de sus vidas en las organizaciones» (Flórez, 2005). Para los docentes, el éxito es la satisfacción personal de realizar un buen trabajo.

Por otro lado, respecto al compromiso/identificación que establece el docente con la institución, el aporte que hace cada miembro de la organización a su propia institución está determinado por el tipo de compromiso que establece, lo cual deviene en un tipo de involucramiento con el puesto de trabajo. Este surge de un vínculo emocional o intelectual que liga al individuo con la organización.

Según Davenport (2006), los tipos de compromiso que se establecen son tres. Primero, el compromiso de actitud que se produce cuando las personas se identifican con la institución, se implican en sus actividades y disfrutan perteneciendo a ella (deseo de pertenecer). Luego, el compromiso programático es aquel que induce a los empleados a permanecer en la institución porque no pueden permitirse abandonarla, debido a los costes económicos o culturales que le generaría (me costará si no pertenezco). Finalmente, el compromiso basado en la lealtad se produce cuando el docente se siente ligado a la institución por sentido de obligación y lealtad, desean hacer lo que consideran oportuno y beneficioso para la organización. Este compromiso está asociado con una intensa motivación, una asistencia consecuente y una dedicación notable a los objetivos institucionales (debo pertenecer).

Las personas con una gran involucración con su puesto de trabajo, es decir, con un compromiso, se dedican e invierten horas en él sin importarle tanto dónde lo realizan y el esfuerzo que ponen en ello. El compromiso con la organización y la involucración con el puesto de trabajo son los puntos focales de la inversión de capital humano.

En conclusión, de acuerdo a Davenport (2006), una eficiente gestión del capital humano implica necesariamente el desarrollo de cada una de las estrategias mencionadas, y un compromiso basado en la lealtad; por ello, en la investigación, se analizó la información a la luz de esta teoría.

\section{Metodología}

La investigación es exploratoria, ya que se examina un tema de investigación poco estudiado en el campo de la educación superior. En esta, se analizan las 
estrategias de gestión del capital humano que se desarrollan en la Facultad de Educación de una universidad particular en Lima.

Se utilizó el modelo de enfoque mixto, que se caracteriza por contener aspectos del enfoque cualitativo y cuantitativo, que, a decir de Hernández (2003), «constituye el mayor nivel de integración entre los enfoques cualitativo y cuantitativo, donde ambos se combinan durante todo el proceso de investigación» (p. 22).

La parte cuantitativa de la investigación comprendió el análisis estadístico de las encuestas en el tratamiento de la información. La otra parte consistió en el análisis cualitativo de las entrevistas realizadas a los directivos de la institución. Al respecto, Sandín y Paz (2003) manifiestan que las investigaciones cualitativas son descripciones detalladas de situaciones que rescatan experiencias, actitudes, pensamientos, y reflexiones, tal y como son expresadas por los entrevistados.

Cabe recalcar que esta investigación no presenta hipótesis, puesto que es un estudio mixto, y, a decir de Hernández (2003), pueden tener o no hipótesis. Su naturaleza es inductiva y su alcance, exploratorio.

Se pretende abrir un camino en esta línea para proseguir con estudios futuros, ya que son pocas las investigaciones que se han realizado sobre el tema en el campo educativo (solo en la educación básica), y en el país no existen investigaciones al respecto. "Los estudios exploratorios se efectúan normalmente, cuando el objetivo es examinar un tema o problema de investigación que no se ha abordado antes» (Hernández, 2003, p. 115).

\subsection{Población y muestra}

La población estuvo conformada por los directivos y docentes de la especialidad de Lengua y Literatura, con más de diez años de servicio en la Facultad de Educación de una universidad privada. Se escogió la presente población debido a la accesibilidad para la aplicación de los instrumentos en el recojo de información.

El tipo de muestra es no probabilística intencional, debido a que responde a un esfuerzo deliberado de obtener una muestra representativa mediante la inclusión de un grupo típico, en este caso los cuatro directivos y siete docentes que se seleccionaron directamente de la población. Se parte del supuesto de que son las personas que pueden dar su perspectiva sobre el tema de estudio y poseen las características relacionadas al fenómeno que se desea estudiar (Vieytes, 2004). 
La muestra no probabilística de los docentes está compuesta por un total de siete catedráticos; cuatro docentes principales y tres docentes contratados. Ambos grupos tienen como característica común que cuentan con más de diez años de servicio en la institución, por lo que han podido vivenciar diferentes gestiones en la organización, característica importante.

La muestra no probabilística intencional de los directivos comprende un total de cuatro gestores: Decano, Secretaria académica, Director de Grados y Títulos, Directora de Educación a Distancia. Los directivos seleccionados son los que definen las estrategias de gestión del capital humano en la Facultad, por ello se les escogió.

\subsection{Técnicas y recogida de información}

Se utilizó la entrevista y la encuesta. Para las interrogantes de sus respectivos cuestionarios, se tradujo las variables de la investigación en preguntas concretas que nos proporcionaron información válida y susceptible de ser cuantificada. Estas variables son las estrategias de gestión capital humano planteadas por Daverport (ver tabla 1), así como sus respectivos indicadores. Esto debido a que el principal objetivo de la investigación es identificar las estrategias de gestión del capital humano de la Facultad de Educación que desarrollan los directivos con respecto a los docentes.

Los instrumentos fueron previamente revisados por expertos y luego validados. Mediante la entrevista, se recogió información sobre las percepciones de los directivos con respecto a las estrategias de gestión del capital humano en la Facultad. La importancia de la aplicación de este instrumento radica en que permitió obtener información con profundidad, así como comprobar la veracidad y consistencia de las respuestas debido a la relación que se estableció con el entrevistado.

La entrevista fue semiestructurada, que consiste a decir de Vieytes (2004), en el diseño, implementación y análisis de una pauta de entrevista a un grupo de entrevistados con el objeto de obtener respuestas a preguntas abiertas comparables entre sí. Se utiliza cuando se necesita obtener información en diferentes lugares sobre las mismas dimensiones del problema. El instrumento que se aplicó fue la guía de entrevista que constó de doce preguntas abiertas, con sus respectivas repreguntas, lo que tuvo por finalidad profundizar en diversas categorías importantes para la investigación.

La entrevista se aplicó a cada directivo en su respectiva oficina dentro de la universidad. Cada una fue grabada, y también se tomaron apuntes de las mismas, indicando el lugar, hora y fecha. 
Por otro lado, la encuesta se utilizó para contrastar la información recogida en la entrevista, se recogió opiniones de los docentes con respecto a actitudes, prácticas y sugerencias. Su importancia estriba en que al ser anónima pudo recoger y complementar datos que no habían sido recogidos en la entrevista. En ese sentido, fue importante para la investigación.

Se aplicó dos encuestas: la primera referida a las estrategias de gestión del capital humano y la segunda encuesta relacionada con el tipo de compromiso que el docente manifestaba tener con la institución: compromiso de actitud, compromiso programático y compromiso basado en la lealtad. Para ello, se utilizó el cuestionario: en el primer caso contenía veinticuatro preguntas varias con opciones de respuesta de alternativas múltiple, relacionadas a las diferentes categorías del fenómeno a investigar. En el segundo caso, contenía solo una pregunta con tres alternativas para marcar, cada una relacionada directamente con el tipo de compromiso del docente. En ese sentido, es un cuestionario que busca una información de carácter cualitativo (Rodríguez, 1996).

El cuestionario de la encuesta se aplicó a cada docente en su salón de clase donde se les reiteró la confidencialidad del caso.

\section{ANÁLISIS DE LA INFORMACIÓN}

De la teoría desarrollada respecto al enfoque del capital humano, emergieron las categorías de análisis que permitieron identificar las estrategias de gestión del capital humano que se desarrollan en la institución, así como los tipos de compromiso que tienen

los docentes con la organización. En ese sentido, la información se analizó de acuerdo a las categorías previamente establecidas que a continuación se detallan:

Tabla 1. Categorías de análisis

\begin{tabular}{|c|c|}
\hline Categorías & Concepto \\
\hline $\begin{array}{l}\text { Contratación de las personas } \\
\text { adecuadas }\end{array}$ & $\begin{array}{l}\text { Estrategia para la contratación de docentes con abun- } \\
\text { dantes capacidades, esfuerzo y tiempo. }\end{array}$ \\
\hline $\begin{array}{l}\text { Disposición del clima } \\
\text { institucional }\end{array}$ & $\begin{array}{l}\text { Contempla la creación de un ambiente que estimule la } \\
\text { aportación de capital humano. }\end{array}$ \\
\hline $\begin{array}{l}\text { Constitución del capital } \\
\text { humano de las personas }\end{array}$ & $\begin{array}{l}\text { Procesos para la constitución de capital humano com- } \\
\text { petente: aprendizaje formal, aprendizaje informal y } \\
\text { construir un capital de información. }\end{array}$ \\
\hline $\begin{array}{l}\text { Retención del capital } \\
\text { humano }\end{array}$ & $\begin{array}{l}\text { Actividades dirigidas a conservar a los inversores de } \\
\text { capital humano. Mediante el compromiso y dedicación. }\end{array}$ \\
\hline
\end{tabular}


En el caso de la entrevista que se aplicó a los directivos y la primera encuesta a los docentes, cada pregunta correspondía a las categorías de análisis. En el caso de la segunda encuesta (docentes), los ítems que contenían se referían directamente a cada uno de los tipos de compromiso, ya explicados. En general, se analizó las respuestas de cada pregunta e ítem a la luz de las categorías presentadas.

\section{Resultados}

Los resultados se agruparon de acuerdo a las categorías de análisis. Estos son producto de la triangulación de la información obtenida de los directivos y docentes mediante la entrevista y las dos encuestas.

\subsection{Contratación de inversores de capital humano}

Las respuestas de los docentes y directivos en general, a excepción del decano, demostraron que no se tiene conocimiento de cómo se lleva cabo el proceso de contratación de los nuevos docentes. El 71\% de los docentes expresó desconocerlo. Directivos y docentes aseguraron que el Decano es la persona que lo realiza y controla toda la información.

Como se aprecia, la mayoría de los docentes desconoce cómo se lleva a cabo este proceso. De ello, se infiere la inexistencia de transparencia en la contratación del nuevo personal, aspecto negativo si tomamos en cuenta que la claridad de los procesos es un punto clave en toda gestión del capital humano que pretenda ser de calidad. Además, la información clara y transparente refuerza y aumenta la confianza del trabajador en la institución, lo que a su vez incrementa la inversión.

\subsection{Disposición del clima institucional}

Sobre este punto, los directivos expresaron que no se toman en cuenta los aportes y opiniones de los docentes, no es parte de la gestión. Asimismo, el $71 \%$ de los docentes encuestados manifestó que las reuniones entre directivos y docentes son esporádicas. En lo que sí coincidieron totalmente es en asegurar que los docentes gozan de total autonomía para el desempeńo de su cátedra.

Otro aspecto resaltante es que la mayoría de los directivos manifestó que se reconoce a los docentes por su desempeño profesional. Por el contrario, los docentes expresaron que son muy pocas las veces en que esto sucede, el $42 \%$ de ellos aseguró que solo algunos docentes conocen el sistema de incentivos, el $29 \%$ expresó que nadie lo conoce. En general, el mayor porcentaje coin- 
cidió en que la mayoría de los docentes desconoce el sistema de incentivos en la institución. Directivos y docentes estuvieron de acuerdo en que el único incentivo que existe es el reconocimiento personal.

Otro punto clave es la comunicación horizontal y fluida entre los responsables de la gestión y los docentes. Los directivos manifestaron ser conscientes de que no existe una comunicación fluida entre ellos en las épocas de incertidumbre; sin embargo, en este punto los docentes son más explícitos al asegurar que no existen canales de comunicación fluida ante hechos importantes que acontecen.

En general, las respuestas evidenciaron la insuficiente existencia de actividades que generen un clima propicio para la inversión adecuada de capital humano.

\subsection{Constitución del capital humano}

Con relación a actividades que generen y desarrollen el capital humano, como las capacitaciones, talleres, los directivos y docentes coincidieron al manifestar que sí se realizan, pero, no de manera frecuente, solo dos veces por semestre académico, como máximo. Los docentes encuestados en un 57\% manifestaron que son pocas las veces que se desarrollan. Es decir, se realizan, pero no como parte de un proceso de planificación sobre la base de las demandas de conocimientos de los docentes, sino a criterio de los directivos y/o alguna demanda urgente que se presente.

Con respecto al aprendizaje social, esto es equipos de trabajo llamados comunidades de práctica formadas de manera voluntaria por los docentes para aprender y compartir información, los directivos en general manifestaron desconocer su existencia en la institución. Por el contrario, la mayoría de los docentes expresaron que sí conocen de su existencia, el 57\% aseguró conocer algunas comunidades de práctica. Se deduce, que existen comunidades de prácticas, sin embargo, no están identificadas por los directivos. Ambos grupos: docentes y directivos coincidieron en que estas comunidades no reciben algún apoyo de parte de la institución.

En lo que sí se evidenció una diferencia de apreciación es en lo concerniente al capital de información. La mayoría de los docentes aseguraron que son muy pocas las veces que sus conocimientos son rescatados para ser transformados en material aprovechable por la organización, el 86\% de los docentes expresó que son pocas las veces que sus conocimientos son transformados en material tangible, como en CD, libros, entre otros. Por el contrario, el $90 \%$ de los directivos entrevistados aseguró que, si elaboran $\mathrm{CD}$, manuales 
y demás materiales, con los conocimientos de los docentes. Esto se explicaría porque los materiales que se producen son solo de los docentes ordinarios, que representan un porcentaje minoritario en la Facultad.

\subsection{Retención del capital humano}

En relación con esta estrategia que contempla como un aspecto clave los incentivos que se otorgan a los docentes. La mayoría de los directivos expresó que sí se les otorga incentivos a los docentes que sobresalen en su cátedra, como son los reconocimientos personales y aumento de sus horas lectivas. Sin embargo, los docentes manifestaron mayoritariamente que no existen incentivos en la Facultad, el $71 \%$ expresó no conocer algún incentivo que otorgue la Institución. Aunque un $29 \%$ identificó el reconocimiento personal como único incentivo.

Es decir, el único incentivo conocido por algunos docentes y aceptado por los directivos es el reconocimiento personal y moral a docentes que han sobresalido en su cátedra. Ambos grupos coincidieron que no existe un sistema formalizado de incentivos que funcione como impulsor de la inversión de capital humano en la institución.

En relación con la remuneración económica, los directivos expresan estar conformes con ella, aunque ninguno expresa que le parece atractiva, al igual que los docentes que aseveran que no consideran la remuneración económica atractiva. De acuerdo a las encuestas aplicadas, el $86 \%$ de los docentes opina que la remuneración económica que perciben no es atractiva en comparación con otras que otorgan las demás universidades del país.

\section{Conclusiones}

La Facultad de la Educación de la universidad en cuestión no cuenta con una gestión que priorice el capital humano como elemento fundamental en el desarrollo de la organización. Las estrategias de gestión existentes no consideran a los docentes como inversores de capital humano que representan una ventaja competitiva para la institución en la presente sociedad del conocimiento. Ello es preocupante si toma en cuenta que la única herramienta diferenciadora que le permitirá a las organizaciones enfrentar los numerosos desafíos en este contexto altamente competitivo es el capital humano con el que cuentan (Alles, 2006).

En la primera estrategia de gestión, proceso de contratación de inversores de capital humano, se evidenció que este no es producto de un proceso de 
planificación ni es desarrollado como una estrategia diseñada por los responsables de gestión en la institución. Es una actividad llevada a cabo de acuerdo a los criterios de la autoridad de la unidad académica. Es decir, la incorporación de nuevos docentes a la institución no forma parte de una estrategia articulada y transparente. Al respecto, la contratación de personal idóneo es una exigencia estratégica de gran relevancia, pues supone dotar a la organización del personal competente con el conocimiento, destreza y talento necesarios para una óptima inversión de capital humano. Esto significará una mayor diferencia competitiva para la organización (Davenport, 2006).

Por otro lado, el clima en la organización no dispone de los elementos necesarios para propiciar un escenario adecuado para la inversión de capital humano por parte de los docentes. Debido a que, por un lado, no se establece el vínculo entre el aporte individual de cada colaborador con los objetivos y estrategias macro de la institución (alineación estratégica). Asimismo, no existe un conocimiento cabal de las competencias de los docentes a fin de ver como cada uno puede contribuir efectivamente con el desarrollo de la organización. Tampoco, existe una valoración de sus aportes en la gestión y no se cuenta con un sistema de incentivos formalizado para los docentes que aliente su inversión de capital humano.

Lo positivo en este aspecto es que sí se les otorga libertad a los docentes para el ejercicio de su cátedra. Al respecto, Davenport (2006) manifiesta que cuando las personas se atienen a unas normas que respaldan la confianza y exigen una menor administración formal, la inversión de capital humano determina un beneficio superior.

Respecto al desarrollo y constitución del capital humano, la Institución carece de un plan de capacitación y formación a los docentes, a fin de perfeccionarlos y actualizarlos en función de las demandas de conocimiento. Las actividades que existen son esporádicas y no forman parte de un programa sobre la base de las necesidades reales del docente, y al contexto actual en la presente Sociedad del Conocimiento. Asimismo, no se incentiva la creación ni permanencia de comunidades de práctica. Todo esto acompañado de las pocas veces que el capital de información es transformado en explícito para su aprovechamiento en la organización. Ello limita la capacidad de la institución de obtener una ventaja competitiva, a través de la habilidad y capacidad para transferir conocimiento entre los miembros de la organización. «La supervivencia de las empresas depende de su capacidad para crear conocimiento en sus recursos humanos y utilizarlos» (Alles, 2006, p. 67)

En otra estrategia clave que se debería desarrollar como es el sistema de incentivos, la institución carece de él como un plan organizado, este es conce- 
bido como un aspecto que debe funcionar cuando los docentes mejoren su desempeño. Es decir, no existen elementos que funcionen como un sistema de incentivos que aliente, mejore o maximice la inversión de capital humano. $\mathrm{Al}$ respecto, cabe recordar que factores como el sueldo, incentivos y oportunidades de desarrollo influyen positivamente en las percepciones de los individuos sobre el grado en que la institución los aprecia y valora, y, por consiguiente, evita la disminución en la inversión de capital humano, es más lo incrementa (Davenport, 2006).

Asimismo, los docentes en mayor porcentaje perciben a la Facultad como una institución que no valora y estimula el capital humano existente. En general, perciben que sus aportes no son tomados en cuenta, y que su labor no es bien remunerada ni incentivada.

Un aspecto resaltante es que, a pesar de lo mencionado, el cien por ciento de los docentes manifiesta tener un compromiso de actitud con la organización (se implican en sus actividades y se sienten identificadas con ella) debido a las relaciones empáticas que han establecido con docentes y alumnos. Lo cual es rescatable y refleja el gran poder de los vínculos emocionales que se establecen en la organización, tomando en cuenta la deficiente gestión del capital humano que existe «El clima organizacional tiene efectos muy poderosos tanto para el desempeńo docente como para los comportamientos de las personas al interior de las organizaciones» (Alvarado 2008, p. 95).

Finalmente, es evidente que esta investigación abre líneas para seguir indagando respecto a cuáles otros elementos permiten fidelizar a un docente con la institución donde labora, a pesar de que las estrategias de gestión no sean efectivas.

\section{REFERENCIAS BIBLIOGRÁFICAS}

Alvarado, O. (2008). Gerencia y marketing educativo. 2da edición. Lima: USIL, Fondo editorial.

Alles, M. (2006). Dirección estratégica de recursos humanos, Gestión por Competencias. 2da edición, Buenos Aires: Granica.

Dădârlat, A. y Dumitraşcu, D. (2015). The role and importance of human capital in contemporary organizations. Review of Management \& Economic Engineering. 14(1), 78-86.

Davenport, T. (2006). Capital humano. Barcelona: Gestión 2000.

Farace, S. y Mazzotta, F. (2015). The effect of human capital and networks on knowledge and innovation in SMEs1. Journal of Innovation Economics \& Management, 16(1), 39-71. https://doi.org/10.3917/jie.016.0039 
Friedman, B., Hatch, J., Walker, D. M. y Morales, E. M. (2000). Atraer, gestionar y retener el capital humano. Barcelona: Paidós.

Flórez, J. (2005). El comportamiento humano en las organizaciones. Lima: Universidad del Pacífico, Fondo editorial.

Hernández, R. (2003). Metodología de la investigación. 3ra edición. México: Mc Graw Hill.

Pérez, A. (2011). Fundamentos de la gestión del conocimiento en la universidad. Gestión Universitaria, 3(2). Recuperado de http://www.gestuniv.com.ar/ gu_08/v3n2a1.htm

Rodríguez, G. (1996). Metodología de la investigación cualitativa. Granada: Aljibe.

Sandín, E. y Paz, M. (2003). Investigación cualitativa en educación: fundamentos y tradiciones. Madrid: Mc Graw-Hill.

Sáez Vacas, F., García, O., Palao, J. y Rojo, P. (2003). Capital humano (II): Gestión del conocimiento e-learning y modelos sociotécnicos. En Temas básicos de innovación tecnológica en las empresas [Internet]. Madrid: Grupo de Sistemas Inteligentes, Departamento de Ingenierías de Sistemas Telemáticos, Universidad Politécnica de Madrid.

Unesco. (2005). Hacia las Sociedades del Conocimiento. París: Organización Mundial de las Naciones Unidad para la Educación la Ciencia y la Cultura. Recuperado de http//www.unesco.org/publications.

Vieytes, R. (2004). Metodología de la investigación en organizaciones, mercado y sociedad: epistemología y técnicas. Buenos Aires: Las ciencias. 